\title{
Evidence for Keratinocyte Immortalization in High-Grade Squamous Intraepithelial Lesions of the Cervix Infected with High-Risk Human Papillomaviruses
}

\author{
Shirley A. Southern, lan W. McDicken, and Charles S. Herrington \\ Department of Pathology, University of Liverpool, Royal Liverpool University Hospital, Liverpool, United Kingdom
}

SUMMARY: In this study, we demonstrate that expression of cyclin B protein is up-regulated and persists into the upper epithelial layers in parallel with cyclin A expression in high-grade squamous intraepithelial lesions (SIL) infected with human papillomaviruses $16,31,33,51,58,66$, and $67(n=33)$. In contrast, low-grade SIL infected with human papillomaviruses 16,18 , $31,33,39,51,52,56,58$, and $66(n=27)$ show weaker cyclin B expression confined to basal and parabasal cells despite extension of cyclin A and Ki67 expression into superficial cells. Moreover, aneusomy is present in $20 \%$ of the high-grade lesions but in none of the low-grade lesions. The persistent expression of cyclin B in high-grade SIL, and the restriction of aneusomy to high-grade SIL suggest that there is cell cycle progression. In combination with in vitro studies, this provides evidence that high-grade SIL lesions have undergone immortalization. (Lab Invest 2000, 80:539-544).

$C$ yclins, cyclin-dependent kinases (CDK), and CDK inhibitors control cell cycle progression and genomic integrity. They ensure the coordinated passage of cells through the cell cycle and provide surveillance mechanisms or checkpoints before the $S$ and $\mathrm{M}$ phases to prevent abnormal DNA replication (Grana and Reddy, 1995). In vitro, high-risk human papillomaviruses (HPV) disrupt these cellular checkpoints, inducing mitotic defects and karyotypic abnormalities through the interactions of E6 and E7 proteins with the G1/S cell cycle regulators p53 and pRb, cyclins $A$ and $E$, CDK 2 , and CDK inhibitors p21 WAF1/CIP1 and p27 ${ }^{\mathrm{KIP} 1}$ (Galloway and McDougall, 1996; ZerfassThome et al, 1996). Moreover, up-regulation of cdc2associated histone $\mathrm{H} 1$ activity by HPV 16 E6 in transfected primary human keratinocytes results in a decreased vigilance of mitotic checkpoints (Thompson et al, 1997). In a recent in vivo study of low-grade cervical squamous intraepithelial lesions (SIL) (Southern and Herrington, 1998), we reported up-regulation of cyclin $A$, cyclin B and cyclin $E$ expression by both low-risk and high-risk HPV infection. Cyclin D1 was overexpressed in $92 \%$ of lesions infected with low-risk HPV types but was absent in $87 \%$ of lesions infected with high-risk HPV types. The morphological pattern of cyclin expression

Received November 22, 1999.

Supported by grants from Wellbeing and the Royal College of Obstetricians and Gynaecologists (H1/96), the University of Liverpool and Royal Liverpool and Broadgreen Hospitals Trust.

Address reprint requests to: $D r$ r. C. S. Herrington, Department of Pathology, University of Liverpool, Royal Liverpool University Hospital, Daulby Street, Liverpool L69 3GA, United Kingdom. Fax: 44151706 5936; E-mail: c.s.herrington@liv.ac.uk was suggestive of $\mathrm{S}$ phase arrest/prolongation or upregulation of cyclin proteins to affect viral DNA replication. Tetrasomy occurred in lesions infected only with high-risk HPV, providing further evidence that G1/S and G2/M cell cycle checkpoints are abnormal in low-grade SIL infected with high-risk HPV.

In vitro, cellular immortalization induced by transfection of high-risk HPV DNA into primary keratinocytes is accompanied by elevated levels of cyclin B, cyclin A and p34 ${ }^{\text {cdc2 }}$ proteins (Steinmann et al, 1994). Immortalization is also associated with structural chromosome abnormalities (Solinas-Toldo et al, 1997) and activation of telomerase (Steenbergen et al, 1996). Moreover, analysis of naturally occurring lesions has recently demonstrated telomerase activation in almost all invasive cervical carcinomas, in some high-grade SIL, but in no low-grade SIL (Snijders et al, 1998). This suggests that immortalization has occurred in some high-grade SIL but not in low-grade SIL. Therefore, in both low and high-grade SIL, we analyzed: i) the expression of cyclin proteins; ii) the infecting HPV type; and iii) the presence of numerical chromosome abnormalities. In most high-grade SIL, cyclin B expression extended into superficial epithelial cells in parallel with cyclin A expression. In contrast, in lowgrade SIL, cyclin B expression was restricted to the lower part of the epithelium despite the extension of cyclin $A$ expression into the upper epithelial layers. Although tetrasomy of the analyzed chromosomes was present in both low and high-grade SIL, aneusomy (chromosome imbalance) was detected only in high-grade SIL. The combination of cyclin overexpression and chromosome abnormalities identified 
in the high-grade SIL is consistent with in vitro immortalization events and is compatible with recent studies of telomerase activation.

\section{Results}

\section{HPV Analysis}

HPV Typing. A 209bp $\beta$-globin fragment was amplified from all tissues demonstrating adequate DNA quality. All 10 normal biopsies, which contained normal ectocervical squamous epithelium and mature and immature metaplastic squamous epithelia were negative for HPV. HPV DNA was identified in all of the 49 low-grade lesions. Twenty-three of these lesions contained low-risk HPV types [HPV $6(n=11), 11(n=$ 6), $42(n=3), 43(n=2)$ and $44(n=1)]$. Twenty-four low-grade SIL were infected with high-risk HPV types [16 $(n=5), 18(n=2), 33(n=1), 39(n=2), 51(n=$ 2), $52(n=3), 56(n=1), 58(n=5), 66(n=3)]$. Double infections were identified in two specimens: the first contained HPV 6 and 18, and the second contained HPV 18 and 31.

All high-grade lesions contained high-risk HPV types [HPV $16(n=19)$, HPV $31(n=4)$, HPV 33 ( $n=$ 2) and HPV $58(n=1)]$. Dual infections were identified in three specimens: the first contained HPV 16 and 66, the second contained HPV 16 and 31, and the third contained HPV 31 and 51. Direct sequencing of the polymerase chain reaction (PCR) product from one lesion that did not hybridize with any of the probes identified HPV 67.

HPV Localization. Morphological localization of HPV infection was established using in situ hybridization (ISH) with a cocktail of probes. Further high-stringency ISH using individual probes was used to discriminate between the different HPV infected areas in the dual infections. Probes for HPV types 6, 18 and 31 discriminated between the different infections in the two low-grade SIL containing HPV 6/18 and HPV 18/31. For the high-grade SIL, the HPV 16 probe was used on the HPV 16/66 lesion; HPV 16 and HPV 31 probes were used separately on the HPV 16/31 lesion; and the HPV 31 probe was used on the HPV 31/51 lesion. No HPV ISH signals were observed in normal ectocervical squamous epithelium or in immature or mature squamous metaplasia.

As only high-risk HPV types were identified in the high-grade SIL, the analysis of the cyclin expression and the chromosomes in low-grade SIL was restricted to those infected with high-risk HPV types. In total, 27 low-grade SIL and 33 high-grade SIL infected with high-risk HPV were examined.

\section{Chromosome Analysis}

Tetrasomy of chromosomes $1,3,11,17,18$, and $X$ was identified in nine low-grade SIL [those infected with HPV $16(n=1), 18(n=1), 33(n=1), 52(n=1)$, $56(n=1), 58(n=3)$ and HPV 18/31 $(n=1)]$. In the dual infection, tetrasomy was present only in the area infected with HPV 31. Tetrasomy persisted throughout the full epithelial thickness in eight of these cases; focal tetrasomy involving only the basal epithelial layer was present in the ninth case. Aneusomy was not identified in any of the low-grade lesions.

Eight of the high-grade lesions [those infected with HPV $16(n=4), 31(n=1), 33(n=1), 58(n=1)$ and $67(n=1)$ ] showed tetrasomy of all six chromosomes. Aneusomy was identified in six lesions (Table 1 and Fig. 1, I and J); each contained HPV 16. In three lesions, the overall pattern was tetrasomy with three to five chromosomes and additional relative gain or loss of one to three chromosomes. Three lesions showed disomy of four to five chromosomes and trisomy of one to two chromosomes. Where present, tetrasomy and aneusomy persisted throughout the epithelial thickness. However, chromosomal changes were focal in most lesions; areas of SIL showing no chromosome abnormalities were also present. Moreover, in three lesions infected with HPV 16, the high-grade SIL showed disomy for all chromosomes whereas adjacent low-grade SIL showed tetrasomy. All chromosome abnormalities, as demonstrated by $\mathrm{ISH}$, were confined to HPV infected areas. Adjacent normal squamous epithelium was disomic in all lesions. Similarly, the 10 normal biopsies that contained normal ectocervical squamous epithelium and immature and mature squamous metaplasia were disomic for all chromosomes.

\section{Cyclin Expression}

Nuclear Ki67 expression extended into superficial epithelial cells in 10 of 27 low-grade SIL (37\%), and showed a similar distribution to cyclin A expression in most cases (Fig. 1, A to $\mathrm{H}$ ). This was not significantly different from the high-grade SIL, in which Ki67 expression extended into the superficial layers in 15 of 33 cases $\left(46 \% ; \chi^{2}=0.4 ; d f=1, p>0.05\right)$. Cyclin $A$ expression was present in cell nuclei and, to a lesser extent, in cytoplasm throughout the epithelial layers (Fig. 1, A and C) in 20 of 27 low-grade lesions (74\%). This was not significantly different $\left(\chi^{2}=0.7 ; \mathrm{df}=1, p>\right.$ 0.05 ) from the high-grade lesions (21 of 33; 64\%)(Fig. $1 \mathrm{G})$. Both Ki67 and cyclin A expression was restricted to occasional basal cells in normal epithelium. Cyclin A and Ki67 expression was of similar intensity in both lesion grades.

Cyclin A expression was frequently present in superficial epithelial cells in the low-grade SIL infected

Table 1. Chromosome Numbers in Aneusomic High-Grade SIL

\begin{tabular}{cccccccc}
\hline & & \multicolumn{6}{c}{ Chromosome } \\
\cline { 3 - 7 } Case No. & HPV Type & X & 1 & 3 & 11 & 17 & 18 \\
\hline 6 & 16 & 6 & 6 & 6 & 4 & 4 & 4 \\
9 & 16 & 4 & 4 & 6 & 4 & 4 & 4 \\
11 & 16 & 2 & 2 & 3 & 2 & 2 & 2 \\
12 & 16 & 2 & 3 & 2 & 2 & 2 & 2 \\
15 & 16 & 6 & 4 & 4 & 4 & 4 & 4 \\
26 & 16 & 3 & 2 & 3 & 2 & 2 & 2 \\
\hline
\end{tabular}



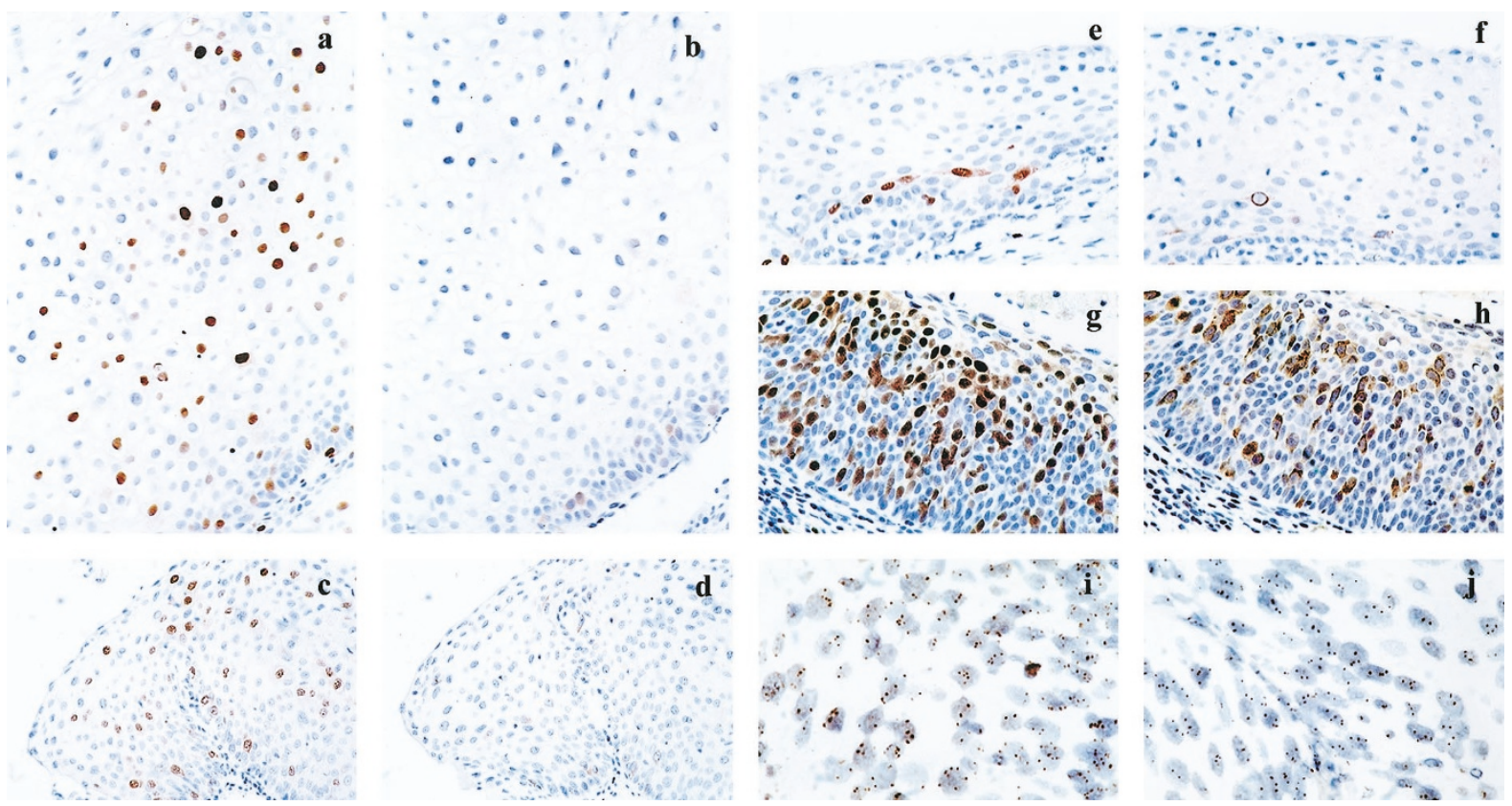

\section{Figure 1.}

Representative sections from two low-grade SIL containing HPV 6 (A and B) and HPV 16 (C and D), and a high-grade SIL infected with HPV 16 (G and H), showing expression of cyclin $A(A, C$, and $G$ ) and cyclin $B(B, D$, and $H)$. The expression of cyclin $B$ extends further up the epithelium and is more intense in the high-grade lesion, whereas the cyclin $A$ distribution is similar in both lesions. Cyclin $A(E)$ and $B(F)$ expression is confined to basal/parabasal cells in immature squamous metaplasia. Aneusomy for chromosome X (I) and chromosome 17 (J) in a high-grade SIL infected with HPV 16. Note the imbalance between the chromosomes. Magnification $\times 400(\mathrm{~A}$ to $\mathrm{H})$ and $\times 630(\mathrm{I}$ and $\mathrm{J})$.

with high-risk HPV (see above). However, cyclin B expression did not extend beyond the lower intermediate layer in any of the lesions. In contrast, in the high-grade SIL cyclin B expression frequently extended into the upper epithelial layers (26 of 33; 79\%). Moreover, cyclin B expression paralleled cyclin A expression in most of the lesions (24 of 33; 73\%) (Fig. $1, G$ and $H)$. The proportion of lesions in which the distribution of cyclin A expression matched cyclin B expression was significantly higher $\left(\chi^{2}=22.8\right.$; $\mathrm{df}=1$, $p<0.001)$ in high-grade lesions than in low-grade lesions ( 3 of $27 ; 11 \%$ ) (Fig. 1, A to D versus Fig. 1, G and $H$ ). The intensity of cyclin $B$ expression also differed between low-grade and high-grade SIL. Lowgrade SIL and adjacent normal epithelium showed similar intensities while high-grade SIL were more intense (Table 2). In normal ectocervical squamous epithelium and immature and mature squamous metaplasia, cyclins $A$ and $B$ were confined to basal and parabasal cells (Fig. 1, E and F). There was no differ-

Table 2. Signal Intensity of Cyclin B Expression Scored from $1+=$ Weak to $4+=$ Very Strong

\begin{tabular}{crrcc}
\hline \multirow{2}{*}{ Lesion } & \multicolumn{4}{c}{ Signal Intensity } \\
\cline { 2 - 5 } & + & ++ & +++ & ++++ \\
\hline Low grade SIL & 12 & 13 & 2 & 0 \\
\hline High grade SIL & 0 & 0 & 2 & 31 \\
\hline
\end{tabular}

The intensity of cyclin $B$ expression differed significantly between low and high grade SIL $\left(\chi^{2}=52.4 ; \mathrm{df}=1, p<0.001\right.$ for $1+/ 2+$ versus $\left.3+/ 4+\right)$. ence in cyclin distribution or signal strength between areas of immature squamous metaplasia, mature squamous metaplasia, or ectocervical squamous epithelium. The major differences between low-grade and high-grade SIL were in the relative distributions of cyclin A and cyclin B expression and in the intensity of cyclin B expression. These differences were clearly demonstrated in high-grade SIL with adjacent lowgrade SIL.

Cyclin D1 expression was absent in 23 of the 27 $(85 \%)$ low-grade SIL and 30 of the $33(91 \%)$ highgrade SIL. Four low-grade lesions (containing HPV 18, 39,52 and 66) and three high-grade lesions (containing HPV 31, 33 and 58) showed persistent cyclin D1 expression in the basal and parabasal region. Weak basal/parabasal expression was present in the normal controls.

In low-grade SIL, nuclear cyclin E expression was present only in the intermediate layers. However, of the 33 high-grade SIL, 9 (27\%) showed cyclin E expression in the intermediate and parabasal layers and $2(6 \%)$ showed expression in the intermediate and superficial cells, whereas in 6 (18\%), cyclin E was present in the parabasal, intermediate and superficial layers. Four of the six lesions with cyclin E expression in the parabasal, intermediate and superficial layers were aneusomic. In the remaining 16 high-grade SIL, cyclin $\mathrm{E}$ expression was present only in the intermediate layers, the same distribution as found in the low-grade SIL. No difference in signal intensity between lesions was noted. Normal ectocervical squamous and metaplastic squamous epithelia showed 
either weak cyclin E expression in occasional intermediate cells or no cyclin E expression.

\section{Discussion}

Cyclin and Ki67 expression were similar in high-grade and low-grade SIL infected with high-risk HPV, with the exception of cyclin B expression and, to a lesser extent, cyclin E expression. In high-grade SIL, cyclin B expression was upregulated and persisted into the upper epithelial layers, in parallel with cyclin A expression. In contrast, in low-grade SIL, cyclin B expression was confined to the basal and lower intermediate cell layers while cyclin A expression extended into superficial keratinocytes. This is consistent with either S-phase prolongation/arrest or specific up-regulation of these cellular proteins. These observations suggest that cells within high-grade SIL continue to cycle and are therefore susceptible to further genetic alteration. Cyclin $A$ and $B$ expression in immature and mature metaplastic squamous epithelia was confined to basal/parabasal cells, indicating that up-regulation of these cyclins in the upper epithelial layers in highgrade SIL did not simply reflect a lack of epithelial maturation.

Although the mechanisms involved in cyclin B regulation are not fully understood, recent evidence has shown that p53 regulates a $G_{2}$ checkpoint through cyclin B; p53 prevents G2/M transition by decreasing cyclin B protein levels and attenuating the activity of the cyclin B promoter (Innocente et al, 1999). Therefore, the increased cyclin B expression observed in our lesions could be due to abrogation of functional p53 by high-risk HPV E6 oncoprotein. The presence of cyclin E expression in parabasal and superficial cells in some of the high-grade SIL suggests that additional cell cycle dysregulation has occurred because, in low-grade SIL, cyclin E is expressed only in intermediate cells, particularly if koilocytic.

Cell cycle dysregulation, particularly of mitotic checkpoints, is probably of central importance to the acquisition of chromosome abnormalities by HPVinfected lesions. Tetrasomy occurred in both lowgrade and high-grade lesions infected with high-risk HPV, whereas aneusomy was confined to the highgrade lesions. This is consistent with the concept of a tetrasomic intermediate state (Andreassen et al, 1996), but a more detailed analysis of individual lesions shows that the situation is more complex. For example, within individual high-grade lesions, foci showing disomy and tetrasomy were identified, suggesting that chromosome reduplication occurred after progression to a high-grade lesion. Similarly, tetrasomy was identified in low-grade SIL adjacent to disomic high-grade SIL, indicating that even in low-grade SIL which show chromosome reduplication, progression to a highgrade lesion does not require persistence of this abnormality. Moreover, two patterns of aneusomy were demonstrated (ie, with and without duplication) consistent with our previous study of invasive carcinomas (Graham et al, 1998). Interestingly, cyclin E expression was present throughout the epithelium in a majority of aneusomic lesions. This is consistent with the recent observation that overexpression of cyclin $E$ causes chromosome instability in rat embryo fibroblasts and human breast epithelial cells (Spruck et al, 1999).

The immortalization of primary human keratinocytes by HPV DNA is thought to occur in part through binding and inactivation of p53 and pRb by viral oncoproteins. The up-regulation of cyclins $A$ and $E$, which ensures entry into $S$ phase, is also likely to be important. This is consistent with the findings in this study. In vitro, immortalization by HPV is associated with a 5 to 10 fold increase in expression of cyclins A and B (Steinmann et al, 1994). This is consistent with the induction of aberrant cyclin A and B expression, alterations in G2/M checkpoints, and aneuploidy by SV40 in human fibroblasts (Chang et al, 1997). Furthermore, progression to immortality of HPV 16 and 18 transfected cells results in chromosomal imbalance and activation of telomerase (Solinas-Toldo et al, 1997; Steenbergen et al, 1996). Our in vivo data show that cyclin B expression persists throughout the epithelium in most high-grade SIL. This finding is consistent with the in vitro observations and suggests that the constituent cells are immortalized. This interpretation is further supported by a recent report that activation of telomerase occurs in some high-grade SIL and in almost all invasive carcinomas, but not in lowgrade SIL (Snijders et al, 1998).

The data presented in this study are consistent both with the in vitro properties of individual HPV proteins and with studies of HPV-induced immortalization. In vitro studies have shown that for high-risk HPV: i) the binding of $E 7$ to $R b$ abrogates the G1/S checkpoint (Thomas et al, 1998); ii) HPV E7 binds cyclin A and E (Mclntyre et al, 1996) resulting in prolonged $S$ phase or $S$ phase arrest; and iii) E7 inhibits cyclin E-associated kinase and up-regulates cyclin A (Schulze et al, 1998). These observations are consistent with our findings in low-grade SIL. However, the persistent expression of cyclin B in high-grade SIL suggests that there is cell cycle progression in these lesions and, in combination with in vitro studies, provides evidence that the cells in these lesions have undergone immortalization. This is also consistent with evidence that high-risk HPV early genes can also disrupt mitotic control checkpoints in vitro (Thompson et al, 1997); cells which continue to cycle in the presence of HPV early gene expression would be more likely to acquire further genetic abnormalities and to undergo immortalization. The restriction of aneusomy to high-grade SIL in this study is compatible with this hypothesis. Overall, our findings provide further in vivo evidence that the ability to induce abnormalities of cell cycle control is central to immortalization and transformation by high-risk HPV.

\section{Materials and Methods}

\section{Choice of Specimens}

Forty-nine low-grade SIL and 30 high-grade SIL were identified from the routine diagnostic files of the department of pathology, Royal Liverpool University 
Hospital. Diagnoses were confirmed independently by two gynecological pathologists (CSH and IWM). Ten cervical biopsies from patients with no history of SIL (eight containing immature metaplastic squamous epithelium, eight containing mature metaplastic squamous epithelium, and eight containing normal ectocervical epithelium) were also selected. Metaplastic tissues were analyzed to address the possibility that the differences observed between low-grade and high-grade SIL might be related to a lack of maturation rather than to the lesion grade. Six-micron parallel formalin-fixed paraffin-embedded sections were cut for immunohistochemistry, interphase cytogenetics, and HPV ISH. Three additional sections were cut for HPV PCR typing.

\section{HPV Typing}

HPV type was established as previously described (Southern et al, 1997), using a GP5+/6+ HPV PCR system to amplify a 140-150bp fragment (depending on HPV type) in the L1 gene followed by hybridization with $5^{\prime}$ digoxigenin-labeled oligonucleotide probes to HPV types 6, 11, 16, 18, 31, 33, 35, 39, 42, 43, 44, 45, $51,52,56,58$ and 66 . Type specificity of these probes was verified using plasmid clones and sequenced PCR products. PCO3 and PCO5 primers, which amplify a 209bp $\beta$-globin fragment, were used to assess DNA quality (de Roda Husman et al, 1995).

\section{HPV Sequencing}

PCR products were cut from the agarose gel and purified using QIAquick Gel Extraction Kit (Qiagen Ltd., Studio City, California). Direct sequencing was then performed in a Prism 373 automated sequencer (Applied Biosystems, Inc., Foster City, California) and the sequences were analyzed using the fast $A$ algorithm of the Genetics Computer Group analysis program to search the Genbank and EMBL databases.

\section{HPV Localization}

ISH using a cocktail probe mixture containing digoxigenin-labeled genomic probes for HPV 6, 11, $16,18,31$, and 33 was performed as previously described (Southern et al, 1997). These probes and hybridization conditions allow cross-hybridization to occur with many HPV types (Herrington et al, 1993) thereby establishing viral localization. The stringency of the ISH was increased by altering the posthybridization wash to $50 \%$ formamide in $0.1 \times \mathrm{SSC}$ at $35^{\circ} \mathrm{C}$ to achieve specificity in the specimens containing dual infections detected by PCR. Hela cells and tissues containing known HPV types were used as positive controls.

\section{Interphase Cytogenetics}

Sections were pretreated and hybridized as previously described (Southern et al, 1997), using biotinylated pericentromeric probes (Oncor, Gaithersburg, Maryland) specific for chromosomes 3 (D3Z1), 17 (D17Z1),
18 (D18Z1) and X(DXZ1). Chromosomes 1 (pUC1.77) and 11 ( $p L C 11 A)$ were digoxigenin-labeled by nicktranslation (Life Technologies Inc., Paisley, Scotland). Signals were detected with peroxidase-conjugated antibodies, diaminobenzidine, and hydrogen peroxide. Signal number was determined at a magnification of $\times 630$. Numerical chromosome complement was determined as previously defined and validated (Southern and Herrington, 1996).

\section{Immunohistochemistry}

Parallel 6- $\mu \mathrm{m}$ formalin-fixed, paraffin-embedded sections were deparaffinized in xylene and rehydrated through graded alcohols. Microwave heat treatment in $0.01 \mathrm{M}$ citric acid buffer ( $\mathrm{pH} 6.0$ ) for 15 minutes at $800 \mathrm{~W}$ was required for antigen retrieval. Endogenous peroxidase was blocked using 1.5\% (v/v) hydrogen peroxide in methanol for 10 minutes, followed by $5 \%$ normal goat serum for 20 minutes. Monoclonal antibodies [anti-cyclin A, clone 6E6 (1:25); anti-cyclin B, clone 7A9 (1:20); anti-cyclin D1, clone P2D11F11 (1:20); and anti-cyclin E, clone 13A3 (1:20) (Novocastra, Newcastle, United Kingdom)] were applied for 1 hour at room temperature. The monoclonal anti-Ki67, clone MIB 1 (BioGenex, Finchampstead, United Kingdom) was applied undiluted for 2 hours at $37^{\circ} \mathrm{C}$, as recommended by the manufacturer. The sections were then incubated in biotinylated sheep anti-mouse, followed by horseradish peroxidase-conjugated avidin-biotin complex (Dako, Bucks, United Kingdom). Signals were developed with diaminobenzidine and hydrogen peroxide. Immunohistochemistry was assessed through the evaluation of the morphological distribution of immunoreactive cells within the basal/parabasal, intermediate and superficial layers of the epithelium for each lesion. When signal intensity differed, this was scored on a scale of $1+$ (weak) to $4+$ (very strong).

\section{Statistical Analysis}

Distributions were compared using the $\chi^{2}$-squared test with a significance level of $p<0.05$.

\section{Acknowledgements}

We are grateful to Dr. E.-M. de Villiers (Deutches Krebsforschungszentrum, Heidelberg, Germany), Dr. G. Orth (Institut Pasteur, Paris, France), Dr. Y. Matsukura (National Institute of Health, Tokyo, Japan) and Dr. A. T. Lorincz (DiGene Diagnostics, Maryland, USA) for provision of HPV plasmid clones; and Dr. A. H. N. Hopman (University of Limburg, Maastricht, The Netherlands) for provision of the pUC1.77 and pLC11A clones.

\section{References}

Andreassen PR, Martineau SN, and Margolis RL (1996). Chemical induction of mitotic checkpoint override in mammalian cells results in aneuploidy following a transient tetraploid state. Mutat Res 372:181-194. 
Chang H-TT, Ray AF, Thompson AD, and Schlegel R (1997). Disregulation of mitotic checkpoints and regulatory proteins following acute expression of SV40 large T antigen in diploid human cells. Oncogene 14:2383-2393.

de Roda Husman A-M, Snijders PJF, van den Brule AJC, Meijer CJLM, and Walboomers JMM (1995). Processing of long-stored archival cervical smears for human papillomavirus detection by the polymerase chain reaction. $\mathrm{Br} \mathrm{J}$ Cancer 72:412-417.

Galloway DA and McDougall JK (1996). The disruption of cell cycle checkpoints by papillomavirus oncoproteins contributes to anogenital neoplasia. Semin Cancer Biol 7:309-15.

Graham DA, Southern SA, McDicken IW, and Herrington CS (1998). Interphase cytogenetics evidence for distinct genetic pathways in the development of high-grade squamous intraepithelial lesions and invasive squamous carcinomas of the cervix. Lab Invest 78:289-296.

Grana X and Reddy EP (1995). Cell cycle control in mammalian cells: Role of cyclins, cyclin dependent kinases (CDKs), growth suppressor genes and cyclin-dependent kinase inhibitors (CKIs). Oncogene 11:211-219.

Herrington CS, Anderson SM, Graham AK, and McGee JO'D (1993). The discrimination of high-risk HPV types by in situ hybridization and the polymerase chain reaction. Histochem J 25:191-198.

Innocente SA, Abrahamson JLA, Cogswell JP, and Lee JM (1999). p53 regulates a $G_{2}$ checkpoint through cyclin B1. Proc Natl Acad Sci USA 96:2147-2152.

Mclntyre MC, Ruesch MN, and Laimins LA (1996). Human papillomavirus $\mathrm{E} 7$ oncoprotein binds a single form of cyclin $\mathrm{E}$ in a complex of cdk2 and p107. Virology 215:73-82.

Schulze A, Mannhardt B, Zerfass-Thome K, Zwerschke W, and Jansen-Durr P (1998). Anchorage-independent transcription of the cyclin A gene induced by the $\mathrm{E} 7$ oncoprotein of human papillomavirus type 16. J Virol 72:2323-2334.

Snijders PJF, van Duin M, Walboomers JMM, Steenbergen RDM, Risse EKJ, Helmerhorst TJM, Verheijen RHM, and Meijer CJLM (1998). Telomerase activity exclusively in cervical carcinomas and a subset of cervical intraepithelial neoplasia grade III lesions: Strong association with elevated messenger RNA levels of its catalytic subunit and high-risk human papillomavirus DNA. Cancer Res 58:3812-3818.
Solinas-Toldo S, Dürst M, and Lichter P (1997). Specific chromosomal imbalances in human papillomavirustransfected cells during progression towards immortality. Proc Natl Acad Sci USA 94:3854-3859.

Southern SA, Evans MF, and Herrington CS (1997). Basal cell tetrasomy occurs in low-grade cervical squamous intraepithelial lesions infected with high-risk human papillomaviruses. Cancer Res 57:4210-4213.

Southern SA and Herrington CS (1996). The assessment of intra-tumoural karyotypic heterogeneity by interphase cytogenetics in paraffin wax sections. J Clin Pathol 49:M283M289.

Southern SA and Herrington CS (1998). Differential cell cycle regulation by low- and high- risk human papillomaviruses in low-grade squamous intraepithelial lesions of the cervix. Cancer Res 58:2941-2945.

Spruck CH, Won K-A, and Reed SI (1999). Deregulated cyclin E induces chromosome instability. Nature 401:297-300.

Steenbergen RD, Walboomers JM, Meijer CJ, van der RaaijHelmer EM, Parker JN, Chow LT, Broker TR, and Snijders PJ (1996). Transition of human papillomavirus type 16 and 18 transfected human foreskin keratinocytes towards immortality: Activation of telomerase and allele losses at $3 p$, 10p, 11q and/or 18q. Oncogene 13:1249-1257.

Steinmann KE, Fang Pei X, Stöppler H, Schlegel R, and Schlegel R (1994). Elevated expression and activity of mitotic regulatory proteins in human papillomavirus-immortalized keratinocytes. Oncogene 9:387-394.

Thomas JT, Laimins LA, and Ruesch MN (1998). Perturbation of cell cycle control by E6 and E7 oncoproteins of human papillomaviruses. Papillomavirus Rep 9:59-64.

Thompson DA, Belinsky G, Chang TH-T, Jones DL, Schlegel $\mathrm{R}$, and Münger K (1997). The human papillomavirus - 16 E6 oncoprotein decreases the vigilance of mitotic checkpoints. Oncogene 15:3025-3035.

Zerfass-Thome K, Zwerschke W, Mannhardt B, Tindle R, Botz JW, and Jansen-Durr P (1996). Inactivation of the cdk inhibitor p27KIP1 by the human papillomavirus type 16 E7 oncoprotein. Oncogene 13:2323-2330. 